\title{
METHODS OF ARTIFICIAL INTELLIGENCE - FUZZY LOGIC
}

\author{
BONATO, J.
}

Abstract: Methods of artificial management represent a new approach to problem management in numerous applications in technical systems. The aim of paper is to demonstrate the application of fuzzy logic in the process of management and control of technical systems (Zimmermann, 2001). Program tool "Fuzzy Logic Toolbox" is used in this work. Its functions are called within software package Matlab. Fuzzy logic at the controlled engine speed is applied, input and output variables are selected, fuzzy sets are specified, and their shape is defined through membership function. The value that reflects the linguistic meaning of each fuzzy set is defined. Results enable the value estimation of the output parameters. This leads to the solution of the identified problem, with the help of base of fuzzy rules for observed characteristics which are stored within the model. If the number of input and output variables increases, this influences inference mechanism and solution reliability, through the use of artificial intelligence methods (expert systems, neural network, fuzzy logic, fuzzy, genetic algorithm).

Key words: fuzzy logic, Fuzzy Inference System, expert systems, Matlab
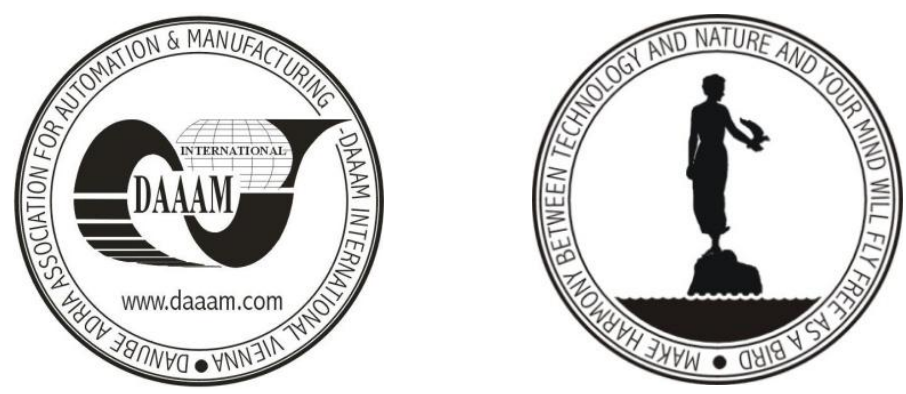

Authors data: Dr. sc. Bonato, J[asminka]; Faculty of Maritime Studies, Studentska 2, 510000 Rijeka, The Republic of Croatia, bonato@pfri.hr

This Publication has to be referred as: Bonato, J[asminka] (2013) Methods of Artificial Intelligence - Fuzzy Logic, Chapter 51 in DAAAM International Scientific Book 2013, pp. 849-856, B. Katalinic \& Z. Tekic (Eds.), Published by DAAAM International, ISBN 978-3-901509-94-0, ISSN 1726-9687, Vienna, Austria DOI: $10.2507 /$ daaam.scibook.2013.51 


\section{Introduction}

Fuzzy logic is a method of artificial intelligence. Its aim is to convert human way of thinking into an algorithm by applying the equivalent mathematical methods. Logic based on fuzzy set is polyvalent, it associates the degree of authenticity to each statement. This is known as the value of membership function (Waterman, 1986).

Nowadays it is used in a very wide range of scientific fields; from social and psychological studies and researches to its application in technology (Altrock, 1995).

Advantages of fuzzy systems are flexibility, wide applicability and tolerance to data imprecision. Furthermore, they are based on linguistic variables and they are applied in classical control problems (ex. Application in turbine regulation and ship steering systems). As a result of this project (Kuljača, 2007), a new model of water turbine was developed, as a software module for Matlab.

The assumption is that if the number of collected input and output data sets of greater quality enlarges, in the amount necessary for a mechanism of fuzzy logic, possibilities of further researches and application of fuzzy logic arise.

\section{Fuzzy Algorithm}

One of the main algorithms of fuzzy logic is Mamdani model, which enables the estimation of specific characteristic stored within the model (Hirota \& Sugeno, 1985). The mechanism of fuzzy inference system - FIS can be demonstrated in four phases: fuzzification, inference, aggregation, defuzzification.

In the process of fuzzification, a number of fuzzy sets is selected for every input variable and their membership functions are defined (Zimmerman, 2001). All linguistic variables with all possible values (sets) fall within if / then rule. Inference is a process in which output variables are fuzzificated in analog way, as was shown in previous phase for input variables. In the process of inference numeric values of variables are converted to linguistic values. Then, a corresponding if/then rule is identified for a specific problem, which gives transferred linguistic value of a variable. Defuzzification phase refers to the process of converting linguistic variable values, obtained in inference phase with the help of membership functions, into numeric values.

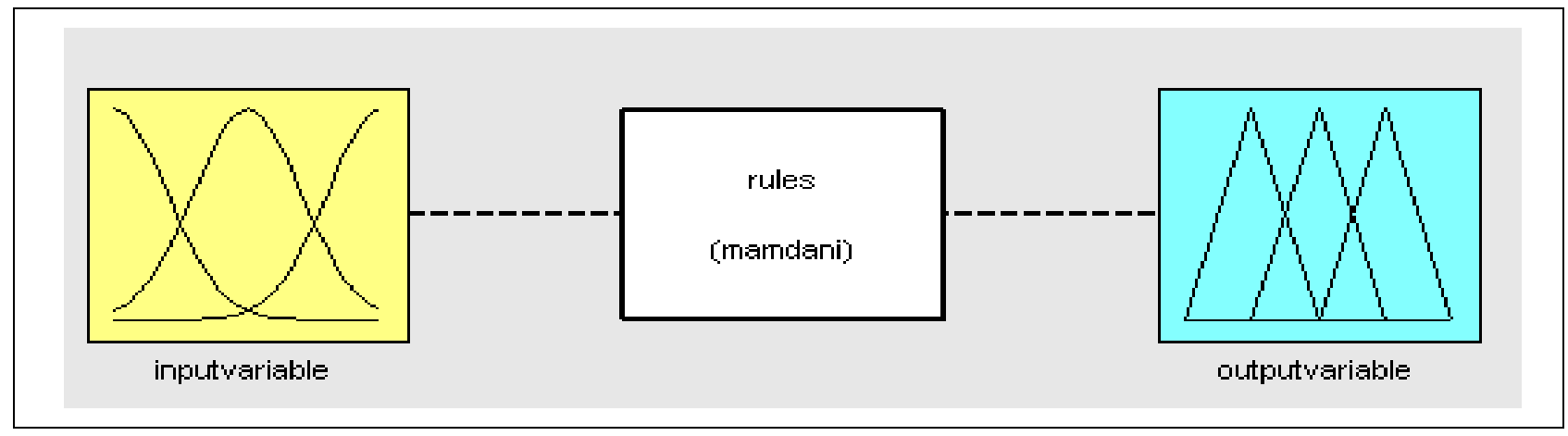

Fig. 1. The structure of fuzzy inference mechanism 


\section{Mathematical Basis of Fuzzy Logic}

Fuzzy logic is based on the theory of fuzzy sets, which can be determined as a generalization of the theory of classical sets. In classical theory sets are clearly defined sets of elements (numbers, symbols, etc), called defined sets.

Elements of all sets observed in a given situation belong to unchangeable, constant set, which is specified as the universal set. In classical theory of membership sets, if $X$ is a universal set and $A$ is an arbitrary set, and if an arbitrary element $x$, from the universal set $X$, does not belong to set $A$, it is defined by characteristic function $\mathcal{X} \cdot \mathrm{X} \rightarrow\{\mathrm{Q}, 1\}$

$$
\chi(x)=\left\{\begin{array}{l}
1, x \in A, \\
0, x \notin A
\end{array}\right.
$$

Therefore, in classical theory, a set is assigned with strictly defined membership; an element either belongs or does not belong to a set. In 1965. Lofti A. Zadeh [6] introduced the term of a fuzzy set, in which more levels of membership were allowed.

Degree of set membership is is marked with values 0 and 1 , as well as with values within the interval $\{0,1\}$.

The change point for fuzzy sets generalizes the evaluation set from the pair of numbers $\{0,1\}$ to all values found in the interval $\{0,1\}$. The starting point for fuzzy stets is generalization of evaluation set from the pair of numbers $\{0,1\}$. Through expanding of evaluation set we change the nature of characteristic function, called membership function, and it is marked with $\mu_{\mathrm{A}}(\mathrm{x})$. We introduce fuzzy sets instead of defined sets. The interval $\{0,1\}$ contains infinite number of values which means that an infinite number of degree of membership is possible. It is said that the function of membership assigns every element of universal set $X$ to interval $\{0,1\}$. This assignment can be noted as follows: $\mu: X \rightarrow[0,1]$.

If an element certainly belongs to set $A$ then $\mu(X)=1$, and if it certainly does not belong to set $A$, then $\mu(X)=0$. If the value falls within the interval $[0,1]$ and if the value differs from 0 and 1 , that number is interpreted as the degree of membership of the element to the set $A$.

When the value approaches to 1 the possibility of membership to the set $A$ increases. If the value approaches to $\mathbf{0}$, possibility of membership to set $A$ reduces.

\section{Application of Fuzzy Logic-engine Rotation Speed Control}

Problem: To control engine rotation speed (ex. from 2400 - 2450 rotations in a minute) depending on the voltage change $(* * *)$

The problem can be solved by applying fuzzy logic (Ross,2004). This means that the measurement of engine speed, provides the data which depends on the time. 
The data can be sorted out as the follows - from the available data it can be inferred that the speed can be: slow, medium or high. That is the input linguistic variable, just as time measuring interval.

Functions of membership represent shaping and quantification of such symbolic subjective descriptions. They are defined according to statistic data or according to criteria specific for the application (Jang, 1995). A program tool Fuzzy Logic Toolbox was used for that purpose, the functions of which are selected within the program package Matlab.

There are several functions of membership in Matlab (Kovačić, 2002), with various shapes (triangular, trapezoid, S- shapes, Fig. 2).
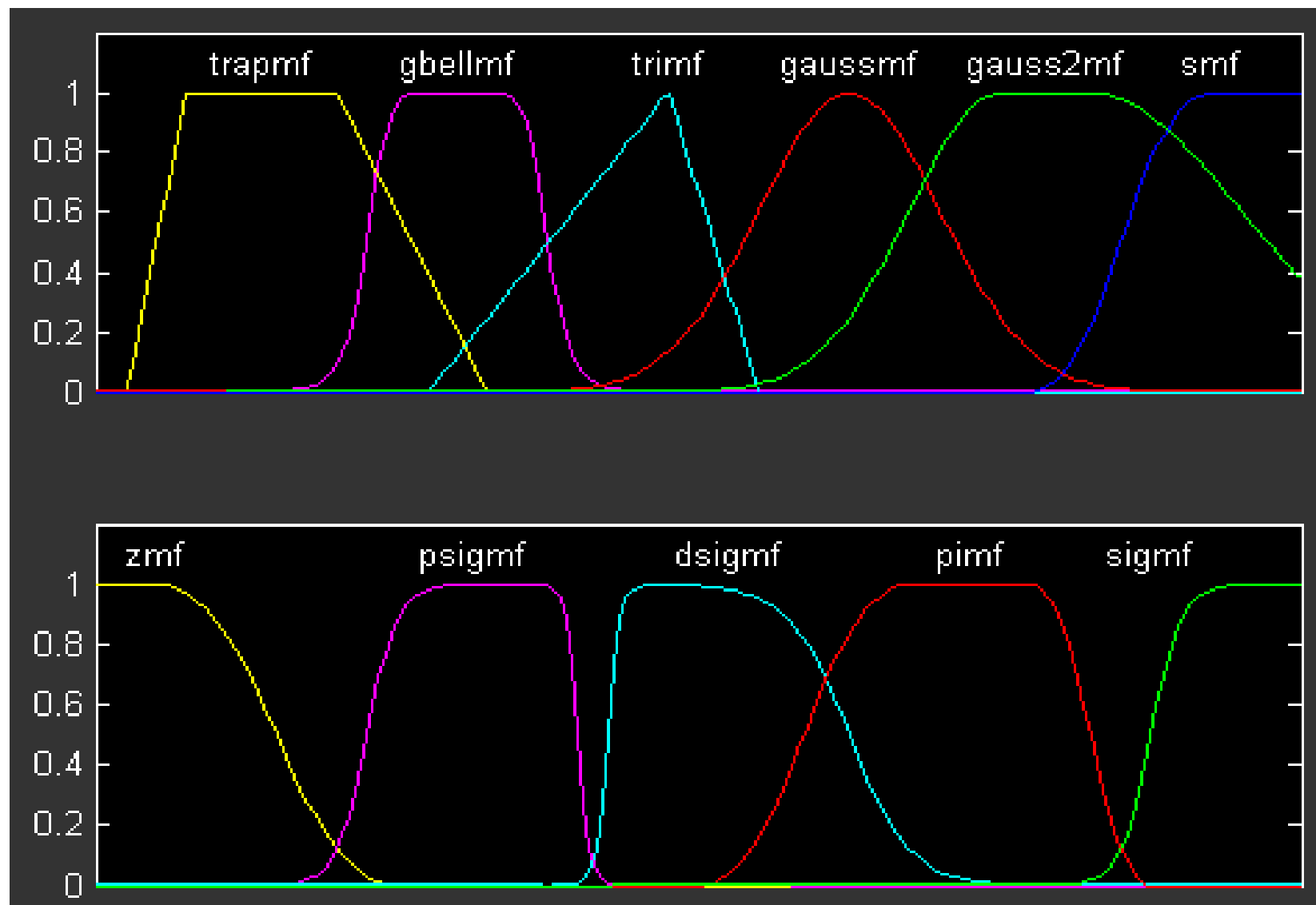

Fig. 2. Shapes of functions of membership

Based upon the overall available data, a certain number of fuzzy sets (Klir \& Yuan, 2010) is defined for each input variable. Their membership functions are defined as well. Precision in engine speed definition in the example depends on the precise choice of membership function (Barett \&Woodall, 1997). For each input variable we define a triangular membership function.

In the second phase (inference) the output variables are fuzzified in an analog way, as was done in the previous phase for the input variables. In this way fuzzy rules are defined. Their shape and number depend on the quality of available data. This is the key phase of the method. In applying these rules, crisp values of all input variables are assigned with as many values of membership functions of each output 
variable as there are the rules defined. Output variable in the example is the engine voltage (low, medium and high).

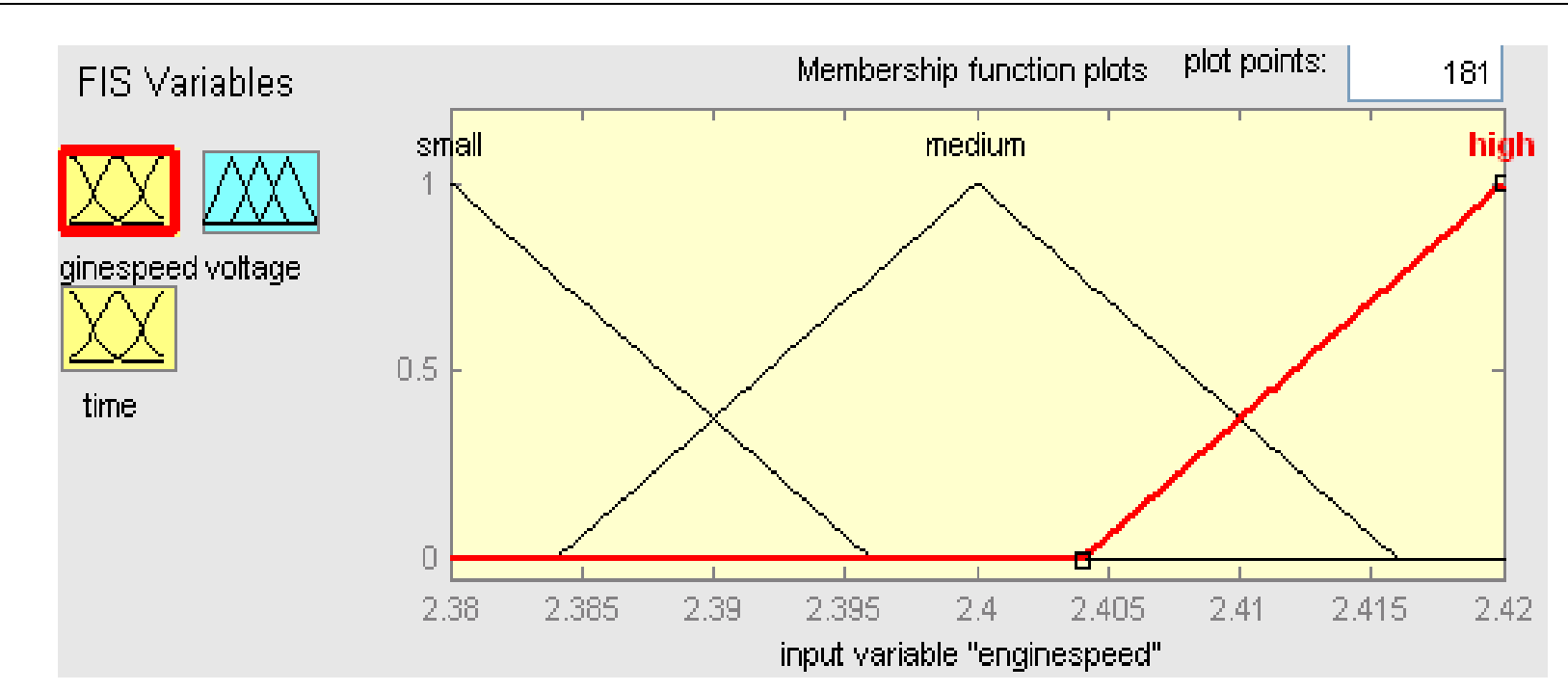

Fig. 3. The shape of the engine speed membership function

1. If (enginespeed is small) then (voltage is small) (1)

2. If (enginespeed is small) and (time is medium) then (voltage is medium) (1)

3. If (enginespeed is medium) and (time is small) then (voltage is medium) (1)

4. If (enginespeed is medium) and (time is small) then (voltage is high) (1)

5. If (enginespeed is high) and (time is medium) then (voltage is high) (1)

6. If (enginespeed is high) and (time is high) then (voltage is high) (1)

7. If (enginespeed is high) and (time is high) then (voltage is high) (1)

8. If (enginespeed is medium) and (time is medium) then (voltage is medium) (1)

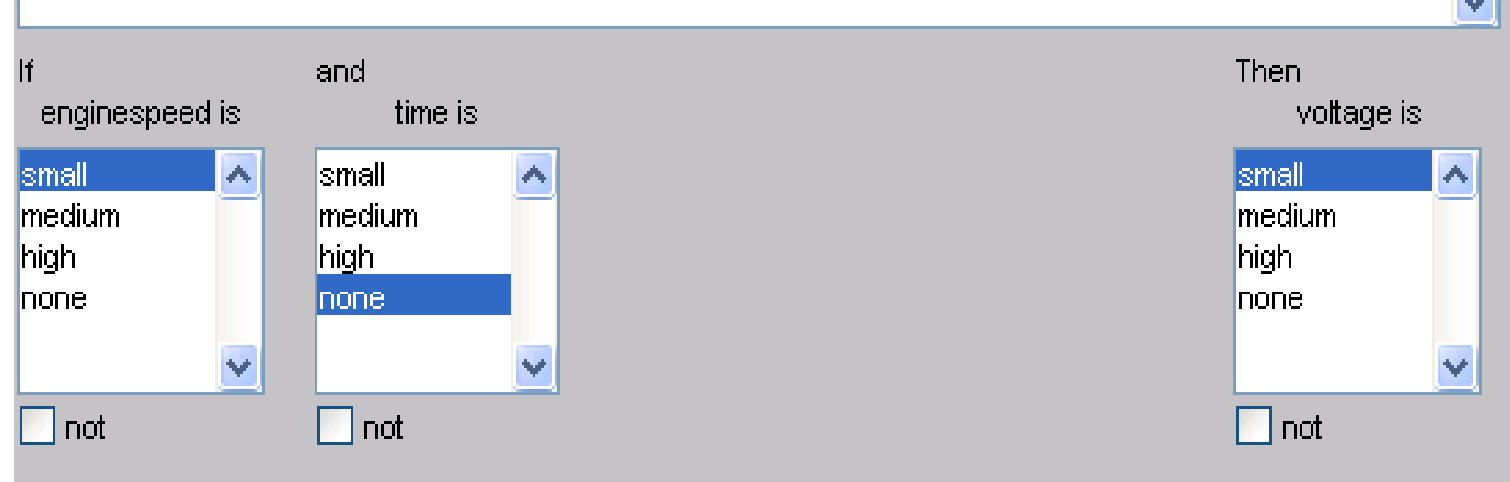

Fig. 4. FIS rules

In the third phase (aggregation), for each variable, the values of membership functions obtained in the previous phase are united. Therefore, the output variable gets one fuzzy set with the defined membership functions.

In the fourth phase (defuzzification), a fuzzy value of each output variable is obtained. 
Bonato, J.: Methods of Artificial Intelligence - Fuzzy Logic

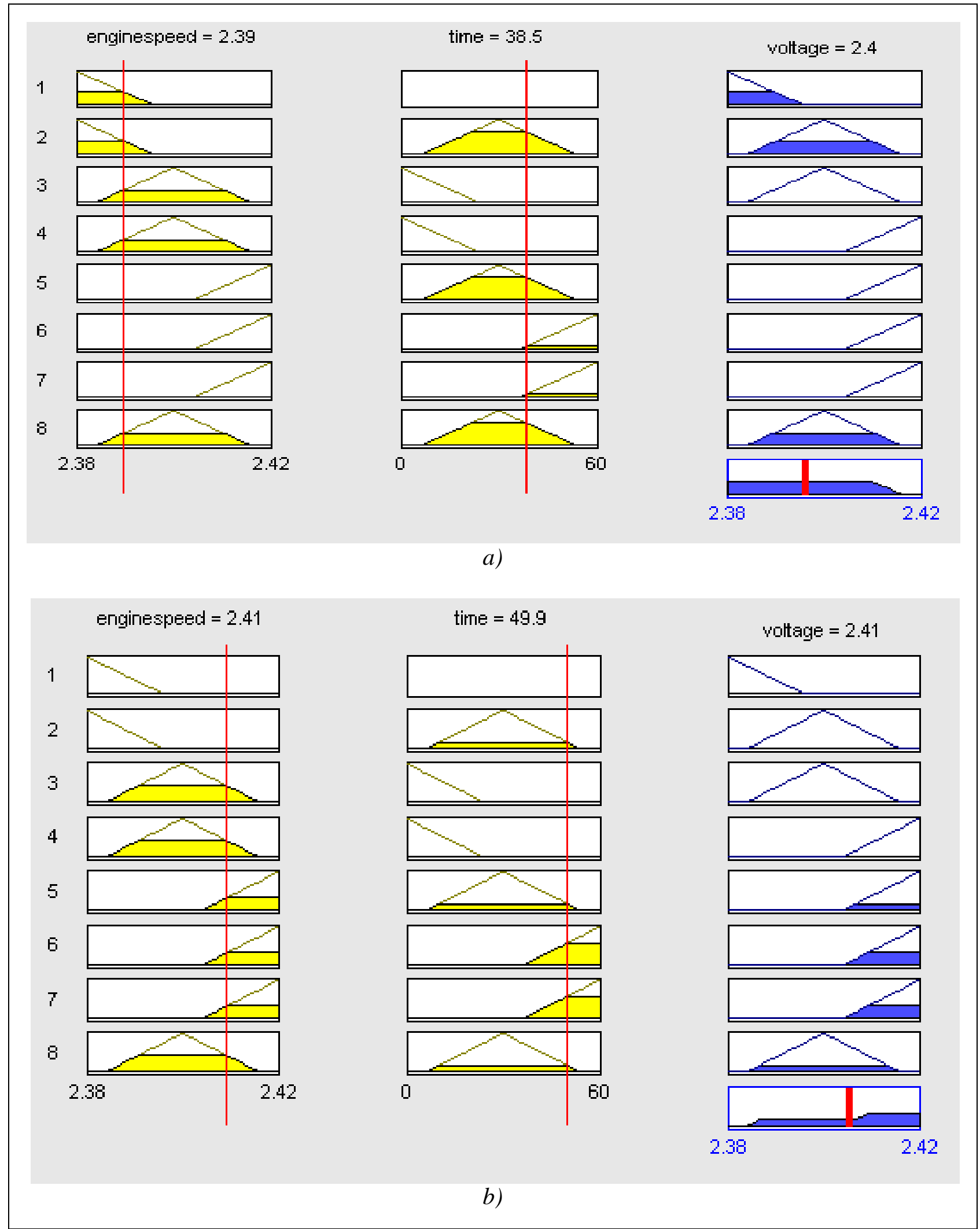

Fig. 5. a) Inference diagram, b) Inference diagram - for the defined input values the expert system calculates the values of output values and gives the graphic scheme 


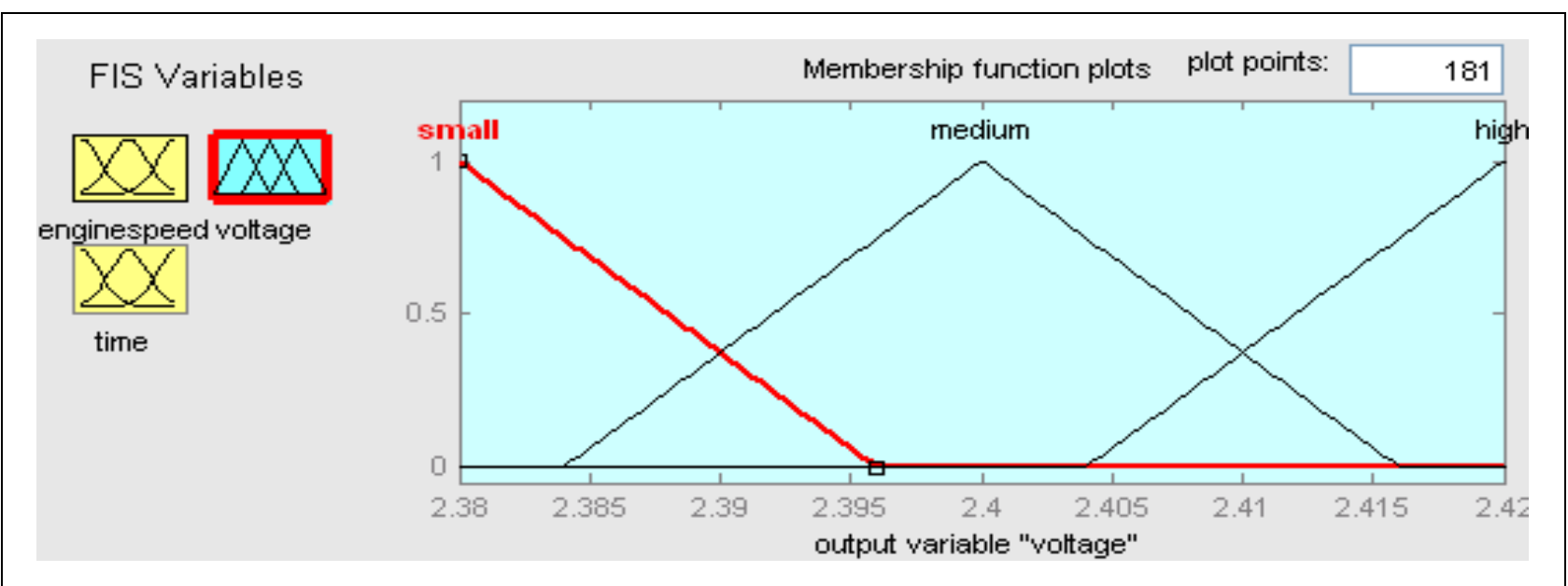

Fig. 6. The shape of membership function of the output variable

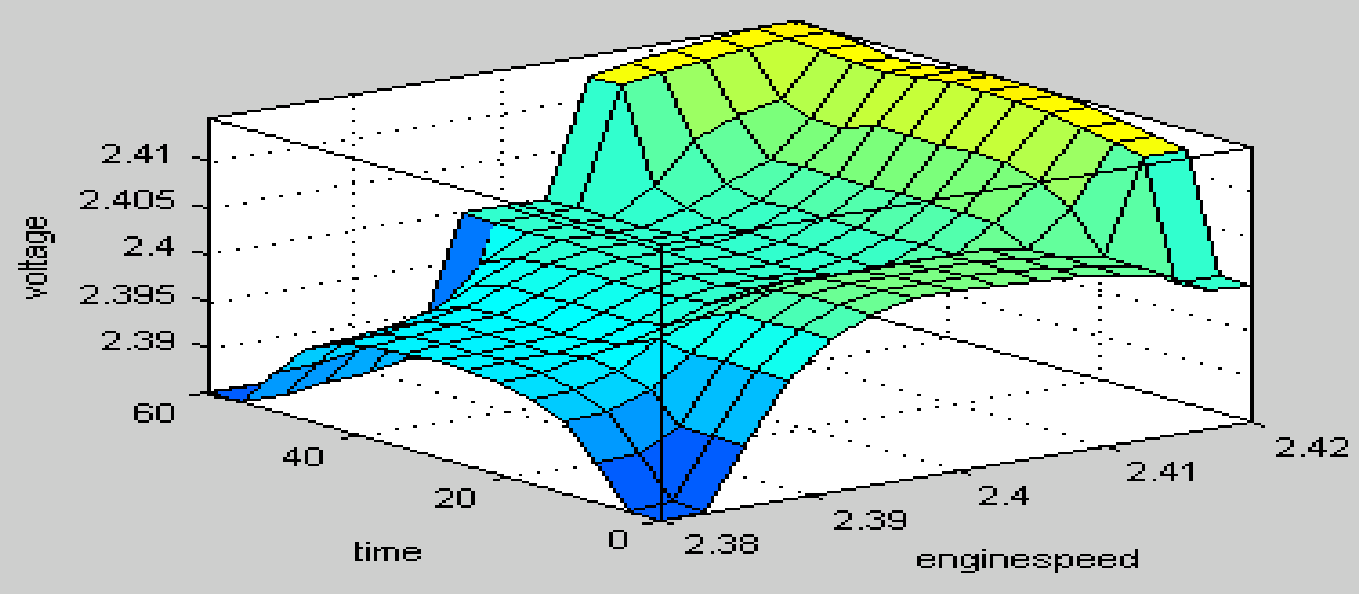

Fig. 7. 3D outline of inference

Defuzzification is a process of converting linguistic results from the database into numeric values. One of the most important characteristic of defuzzification is continuity. The method of fuzzification is continuous if the infinitesimal change of the input variable does not cause a sudden change of any output variable. In this work, The Center of Area method was used. In this method certain fuzzy sets of output linguistic variable are "cut down" for the amount obtained through implication. The areas beneath the membership coefficient for each linguistic set are superimposed into a new fuzzy set (shaded area) and its center of gravity is calculated.

Calculations of numeric values of linguistic output variable are shown in pictures $5 \mathrm{a}$ and $5 \mathrm{~b}$ where the conclusion diagrams are shown (Zadeh, 1996).

\section{Conclusion}

This article analyzes the problem of the influence of engine speed to output voltage in time span of $60 \mathrm{~s}$, with the help of fuzzy logic. FIS is formed for the purpose of tracking the changes in these parameters, as well as for the possibility of 
adjusting shapes and positions of membership functions within a universal set. Membership functions may represent a subjective record of fuzzy pictures ( low voltage for example). They can be defined according to statistic data, however they are not arbitrarily associated, they are based on the criteria specific for the application.

Expert system can calculate the value of output variables, as well as some other numeric values of input variables. It also offers the possibility of presenting them graphically in deduction diagram. Therefore, user can solve the specified problem, on the basis of fuzzy logic rules. For further research it is important to note that fuzzy logic enables the expert automatic problem solving and evaluation of result reliability, by calculating NRMSE parameters for as many input - output variables as possible.

\section{Acknowledgements}

The present work was supported by the Ministry of Science, Education and Sports the Republic of the Republic of Croatia (Research Project 069-0691668-3007), the project leader Julijan Dobrinić. The author wishes to thank Julijan Dobrinić for the cooperation in the development of the research. It is a pleasure to thank Julije Skenderović for interesting discussions, after which parts of this work were written.

\section{References}

Zimmerman, H. J. (2001). Fuzzy set theory and its application,s, Kluwer Academic Publishers, ISBN 0-7293-7435-5, USA

Waterman, D.A. (1986). A Guide to Expert Systems, Addison Wesle Professional, ISBN-10: 0201083132, USA

Altrock C., (1995). Fuzzy Logic and Neurofuzzy Applications

Explained, Prentice Hall PTR, ISBN-10: 0133684652, USA

Kuljača, O. (2007). Applied neuro-fuzzy control of turbine governors and ship rudder systems, Available from: www.hrbi.hr/ukf/work_cro.php-,Accessed: 12.01.2007.

Hirota, K. \& Sugeno, M. (1985). Industrial applications of fuzzy technology in the world, World Scientific Publishing Co. Pte. Ltd., ISBN 9810223668, USA

***Fuzzy Logic Examples using MATLAB,Available from:

http://arri.uta.edu/acs/ee5322/lectures/Ballal\%20fuzzy\%20logic\%20notes.pdf

Ross, T.J.(2004). Fuzzy Logic with Engineering Applications, John Wiley \& Sons, Ltd ISBNs: 0-470-86074-X (HB); 0-470-86075-8, United Kingdom

Roger Jang, J.S., Gully, N. \& MathWorks,Inc. (1987). MATLAB Fuzzy Logic Toolbox: User's Guide, MathWorks, Available from: http:// www.mathworks.com Kovačić,Z. (2002). Intelligent control systems Available from: http://www.fer.unizg.hr/_download/repository/FLT_upute.pdf

Klir,G. J. \& Yuan,B. (1995). Fuzzy Sets and Fuzzy Logic Theory and Applications, Prentice Hall, ISBN-10: 0131011715, USA

Ross, T. J. (2010). Fuzzy logic, John Wiley \& Sons, ISBN 978-0-470-74376-9, United Kingdom 